\title{
CAMP alteration of growth rate of Aspergillus fumigatus and Aspergillus niger is carbon- source dependent
}

\author{
Brian G. Oliver, John C. Panepinto, David S. Askew and Judith C. Rhodes
}

Author for correspondence: Judith C. Rhodes. Tel: +1 513558 0130. Fax: +1 5135582289.

e-mail: judith.rhodes@uc.edu

Department of Pathology and Laboratory Medicine, University of Cincinnati, Cincinnati, OH 45267-0529, USA

\begin{abstract}
CAMP signalling has been shown to be essential for normal growth, morphology and virulence in fungal pathogens of both plants and animals. The effects of exogenous CAMP on the growth of the opportunistic pathogen Aspergillus fumigatus were compared to those of Aspergillus niger, which has previously been demonstrated to respond to extracellular CAMP. Both CAMP and phosphodiesterase inhibitors markedly reduced the radial growth rate of A. niger after $48 \mathrm{~h}$ on minimal medium with glucose as the carbon source, whereas the growth of $A$. fumigatus was not affected by CAMP. However, when glycerol, which does not initiate carbon catabolite repression, was used as a carbon source, cAMP inhibited the radial growth rate of only A. fumigatus $(P<0.05)$. The addition of CAMP to glycerol-minimal medium resulted in a fourfold increase in protein kinase $A$ activity in $A$. fumigatus cell extracts when compared with pre-treatment samples. The protein kinase $A$ activity in $A$. fumigatus cell extracts from cultures grown in glucose did not change significantly with the addition of CAMP. These studies demonstrate that although the growth rates of both $A$. fumigatus and $A$. niger are sensitive to the addition of exogenous CAMP, the response of each organism is distinct and dependent on the carbon source used.
\end{abstract}

Keywords: PKA, phosphodiesterase inhibitor, glucose repression

\section{INTRODUCTION}

The ability of organisms to respond to extracellular stimuli is essential for survival. The cAMP/protein kinase A (PKA) signalling pathway has been demonstrated to be critical in detecting environmental changes and translating those changes into a biological response in many organisms (Thevelein, 1994). Upon receptor activation, a receptor-coupled G-protein activates adenyl cyclase. Adenyl cyclase catalyses the conversion of ATP to cAMP, which accumulates in the cell, leading to activation of PKA (Lengeler et al., 2000). PKA is a heterotetrameric molecule composed of two regulatory subunits and two catalytic subunits. cAMP binds to the regulatory subunits, which causes a conformational change that releases the catalytic subunits to autophosphorylate one another and to phosphorylate downstream targets (Taylor \& Radzio-Andzelm, 1994). The cAMP/PKA pathway has been demonstrated to play an

Abbreviations: IBMX, 3-isobutyl-1-methylxanthine; PDE, phosphodiesterase; PKA, protein kinase A. important role in response to environmental stress, particularly nutritional stress, in the model organism Saccharomyces cerevisiae.

The cAMP/PKA pathway of $S$. cerevisiae has been proposed as the mechanism by which the cell detects changes in glucose availability and adjusts its metabolic machinery accordingly. To maintain metabolic efficiency, the presence of glucose in the medium stimulates the PKA pathway, which leads to repression of genes involved in metabolizing non-glucose carbon sources (Thevelein, 1994). Cells with increased PKA activity, due to deletion of the PKA regulatory subunit, exhibit an inability to grow on non-fermentable, non-repressing carbon sources such as glycerol and ethanol, as well as the weakly fermentable, weakly repressing carbon source galactose (Toda et al., 1987). These mutants also fail to sporulate in response to nutritional stress (Malone, 1990). Mutants with reduced PKA activity express genes normally associated with growth on nonglucose carbon sources, and they sporulate on rich medium, which usually suppresses sporulation in wildtype cells (Casperson et al., 1985). The effects of PKA 
signalling have also been studied in Aspergillus species. In Aspergillus niger, strains that overexpress the catalytic subunit of PKA exhibit a slower growth rate relative to wild-type cells (Bencina et al., 1997). They display a similar phenotype to that of wild-type A. niger grown on glucose-containing medium supplemented with cAMP (Bencina et al., 1997). However, strains of Aspergillus nidulans that overexpress the catalytic subunit of PKA exhibit no alterations with regard to growth rate when grown on medium containing glucose (Shimizu \& Keller, 2001). Although features of PKA signalling have been reported in these two Aspergillus species, the PKA pathway in Aspergillus fumigatus has not been studied.

A. fumigatus is an opportunistic pathogen of humans that plays a key role in the compost cycle in nature. To grow in the lung, the organism must be able to sense the change in environment and translate that change into an appropriate metabolic response. It has recently been shown that transcript levels of the PKA regulatory subunit are elevated when the fungus grows in the presence of endothelial cells, suggesting that PKA may play a role in signalling that environmental change (Rhodes et al., 2001). This would be compatible with data from the fungal plant pathogens Ustilago maydis, Magnaporthe grisea and Colletotrichum trifolii, as well as the human pathogens Cryptococcus neoformans and Candida albicans, in which PKA signalling has been shown to be required for wild-type virulence (D'Souza et al., 2001; D’Souza \& Heitman, 2001; Kronstad et al., 1998; Kruger et al., 1998; Sonneborn et al., 1999; Xu et al., 1997). Because little is known of the importance of this pathway and the effects it may have on the growth of A. fumigatus, the response of the organism to agonists and antagonists of the cAMP/PKA pathway was studied on media containing different carbon sources.

\section{METHODS}

Isolates and culture conditions. A. fumigatus $\mathrm{H} 237$ and A. niger isolate AS-3 are both clinical isolates. Isolates were maintained on Sabouraud's dextrose agar at $37^{\circ} \mathrm{C}$.

Radial growth assay. Conidia were harvested with sterile distilled water, filtered through Miracloth (Calbiochem) and counted in a haemocytometer. Ten microlitres of a conidial suspension $\left(10^{3}\right.$ conidia $\left.\mu \mathrm{l}^{-1}\right)$ were spotted onto Aspergillus minimal medium with $10 \mathrm{mM}$ sodium nitrate as the nitrogen source (ASP MM) (Cove, 1966) plates or on to ASP MM plates containing cAMP, cAMP analogues or phosphodiesterase (PDE) inhibitors. Triplicate plates of all media were inoculated. Indicated carbon sources were used at $1 \%$ final concentrations. cAMP, cAMP analogues and the PDE inhibitors 3-isobutyl-1-methylxanthine (IBMX) and theophylline were used at concentrations between 1 and $10 \mathrm{mM}$. Vehicle controls were performed with the appropriate amount of the respective solvent. Dose-response studies were used to choose suboptimal levels of cAMP and IBMX for experiments in which the compounds were combined to test for additive activity. All chemicals were obtained from Sigma.

Radial colony growth was monitored for cultures of $A$. fumigatus and $A$. niger at $37^{\circ} \mathrm{C}$ every $12 \mathrm{~h}$ for 48 to $60 \mathrm{~h}$.
Colony diameter was measured at each time point with callipers. Growth rates, in $\mu \mathrm{m} \mathrm{h}^{-1}$, were based on colony diameters measured at 24 and $48 \mathrm{~h}$, except for acetate, which used measurements taken at 48 and $60 \mathrm{~h}$. All experiments were performed a minimum of two times, and results from representative experiments are presented.

Enzyme activity assays. Fifty millilitre aliquots of ASP $\mathrm{MM}+1 \%$ glucose were inoculated with $1 \times 10^{8}$ conidia and cultures were incubated at $37^{\circ} \mathrm{C}, 225$ r.p.m., for $12 \mathrm{~h}$. Because glycerol does not support robust germination, conidia were allowed to germinate in ASP MM $+1 \%$ glucose for $6 \mathrm{~h}$ before being washed twice with ASP MM $+1 \%$ glycerol and transferred to $50 \mathrm{ml} \mathrm{ASP} \mathrm{MM+1 \%} \mathrm{glycerol.} \mathrm{These} \mathrm{cultures} \mathrm{were}$ then grown at $37^{\circ} \mathrm{C}, 225$ r.p.m. for $12 \mathrm{~h}$. At the end of the growth period, hyphae were treated with cAMP at a final concentration of $10 \mathrm{mM}$ and collected by vacuum filtration after $0,5,15$ or 30 min continued incubation. Hyphae were washed quickly with ice-cold $0.8 \% \mathrm{NaCl}$, frozen in liquid nitrogen, and crushed with a mortar and pestle to obtain cell extracts. Samples were then resuspended in extraction buffer (25 mM Tris/HCl pH 7.4, $10 \mathrm{mM} \mathrm{MgCl}_{2}, 150 \mathrm{mM} \mathrm{NaCl}$, $1 \mathrm{mM}$ DTT, $1 \mathrm{mM}$ EDTA) and incubated on ice for $30 \mathrm{~min}$. The homogenate was centrifuged at $10000 \mathrm{~g}$ for $10 \mathrm{~min}$ at $4{ }^{\circ} \mathrm{C}$, and the supernatant was used in the assay. PKA enzyme activity was measured by kemptide phosphorylation (colorimetric PKA assay, Spinzyme format; Pierce) using the manufacturer's protocol with the following modification: no activator solution $(500 \mu \mathrm{M}$ cAMP) was used in the assay to measure the amount of PKA activity in the extracts. Purified bovine cardiac PKA-C (Pierce) was used as a standard. The amount of phosphorylated product was measured spectrophotometrically at $570 \mathrm{~nm}$ in a 96-well plate and normalized to the amount of total protein used in the assay. Protein was measured using the BCA assay (Pierce) as described by the manufacturer. Triplicate cultures were processed for cell extracts under each experimental condition. Each experiment was repeated and results from representative experiments are shown.

Statistical analysis. Statistical significance was determined by one tailed, paired $t$-test for means with a $95 \%$ confidence interval. Values are expressed as the mean \pm SEM. Statistical tests were performed using Microsoft Excel.

\section{RESULTS AND DISCUSSION}

The cAMP/PKA pathway has been demonstrated to be a critical mediator of growth and response to nutritional stress in S. cerevisiae (Thevelein \& de Winde, 1999). Proper pathway function is also required for virulence in the plant pathogens M. grisea and U. maydis, as well as in the mammalian pathogens Can. albicans and Cry. neoformans (Lengeler et al., 2000). Despite the importance of the cAMP/PKA pathway in the growth, stress response and virulence of fungi, little is known of the function of this pathway in the opportunistic pathogen A. fumigatus.

Previous studies in A. niger demonstrated a reduction in growth when glucose-containing medium was supplemented with cAMP. Likewise, strains of A. niger that overexpress the catalytic subunit of PKA also show growth inhibition (Bencina et al., 1997). To determine whether PKA signalling plays a role in A. fumigatus growth, colonies of $A$. fumigatus were grown on 
Table 1. Growth rate $\left(\mu \mathrm{m} \mathrm{h}^{-1}\right)$ of $A$. fumigatus and $A$. niger when grown on minimal medium with various carbon sources in the presence of CAMP, CAMP analogues and PDE inhibitors

Results were not statistically significant $(P>0 \cdot 05)$ unless otherwise indicated.

\begin{tabular}{|c|c|c|}
\hline \multirow[t]{2}{*}{ Medium } & \multicolumn{2}{|c|}{ Growth rate } \\
\hline & A. fumigatus & A. niger \\
\hline \multicolumn{3}{|l|}{ ASP MM+glucose } \\
\hline Alone & $712 \pm 15$ & $694 \pm 3$ \\
\hline$+10 \mathrm{mM}$ cAMP & $721 \pm 10$ & $128 \pm 6^{*}$ \\
\hline +10 mM Dibutyryl-cAMP & $762 \pm 15$ & $547 \pm 11 \dagger$ \\
\hline +Ethanol $\neq$ & $731 \pm 7$ & $562 \pm 4$ \\
\hline +10 mM 8-Bromo-cAMP & $750 \pm 1$ & $379 \pm 4+$ \\
\hline +1 mM DMSO $\mathbb{S}$ & $707 \pm 11$ & $479 \pm 5$ \\
\hline$+1 \mathrm{mM}$ IBMX & $632 \pm 10 \dagger$ & $414 \pm 4 *$ \\
\hline$+10 \mathrm{mM}$ DMSO & $586 \pm 1$ & $315 \pm 4$ \\
\hline$+10 \mathrm{mM}$ IBMX & $207 \pm 5^{*}$ & $243 \pm 14+$ \\
\hline$+\mathrm{NaOH} \|$ & $701 \pm 6$ & $567 \pm 13$ \\
\hline$+10 \mathrm{mM}$ Theophylline & $236 \pm 1 *$ & $480 \pm 4^{*}$ \\
\hline \multicolumn{3}{|l|}{ ASP MM+glycerol } \\
\hline Alone & $708 \pm 6$ & $418 \pm 6$ \\
\hline+10 mM cAMP & $656 \pm 12 \dagger$ & $401 \pm 6$ \\
\hline +1 $\mathrm{mM}$ DMSOS & $700 \pm 17$ & $386 \pm 4$ \\
\hline$+1 \mathrm{mM}$ IBMX & $615 \pm 15^{*}$ & $332 \pm 6$ \\
\hline +10 mM DMSOS & $492 \pm 9$ & $279 \pm 17$ \\
\hline$+10 \mathrm{mM}$ IBMX & $206 \pm 1^{*}$ & $150 \pm 15 *$ \\
\hline \multicolumn{3}{|l|}{ ASP MM+ acetate } \\
\hline Alone & $386 \pm 22$ & $475 \pm 13$ \\
\hline$+10 \mathrm{mM}$ cAMP & $281 \pm 20 \dagger$ & $407 \pm 11+$ \\
\hline$+0.5 \mathrm{mM}$ IBMX & $369 \pm 19$ & $475 \pm 21$ \\
\hline \multicolumn{3}{|l|}{ ASP $\mathrm{MM}+$ galactose } \\
\hline Alone & $560 \pm 24$ & INS \\
\hline +10 mM cAMP & $470 \pm 12 \dagger$ & Ins \\
\hline$+0.5 \mathrm{mM}$ IBMX & $417 \pm 12 \dagger$ & INS \\
\hline
\end{tabular}

$* P<0 \cdot 001$.

$\dagger P<0 \cdot 05$.

₹ Growth control for 8-bromo-cAMP.

\$ Growth control for IBMX.

\| Growth control for theophylline.

Insufficient growth in $60 \mathrm{~h}$.

minimal medium with different carbon sources, with or without cAMP.

\section{Effects of exogenous cAMP}

A. fumigatus and $A$. niger were grown on glucosecontaining medium supplemented with cAMP to determine the effect of cAMP on growth rate. Although $A$. niger displayed a reduction in growth rate $(P<0.001)$ on medium containing cAMP, as previously demonstrated (Bencina et al., 1997), there was no growth rate reduction seen with A. fumigatus (Table 1 ; Fig. 1). To test the possibility that A. fumigatus was impermeable to cAMP or was degrading the cAMP, the non-hydrolysable and lipid-soluble cAMP analogues 8-bromo-cAMP and dibutyryl-cAMP were used in place of cAMP. Neither cAMP analogue changed the growth rate of A. fumigatus, but the growth rate of A. niger was significantly reduced by both analogues (Table 1 ). The use of PDE inhibitors with intact cells has been reported to result in an increase in intracellular cAMP levels (Beebe \& Corbin, 1986). The addition of either of the PDE inhibitors IBMX or theophylline to the medium led to a reduction in the growth rate of both A. fumigatus and $A$. niger, suggesting either that cAMP and its analogues were not able to enter the cells of A. fumigatus or that 

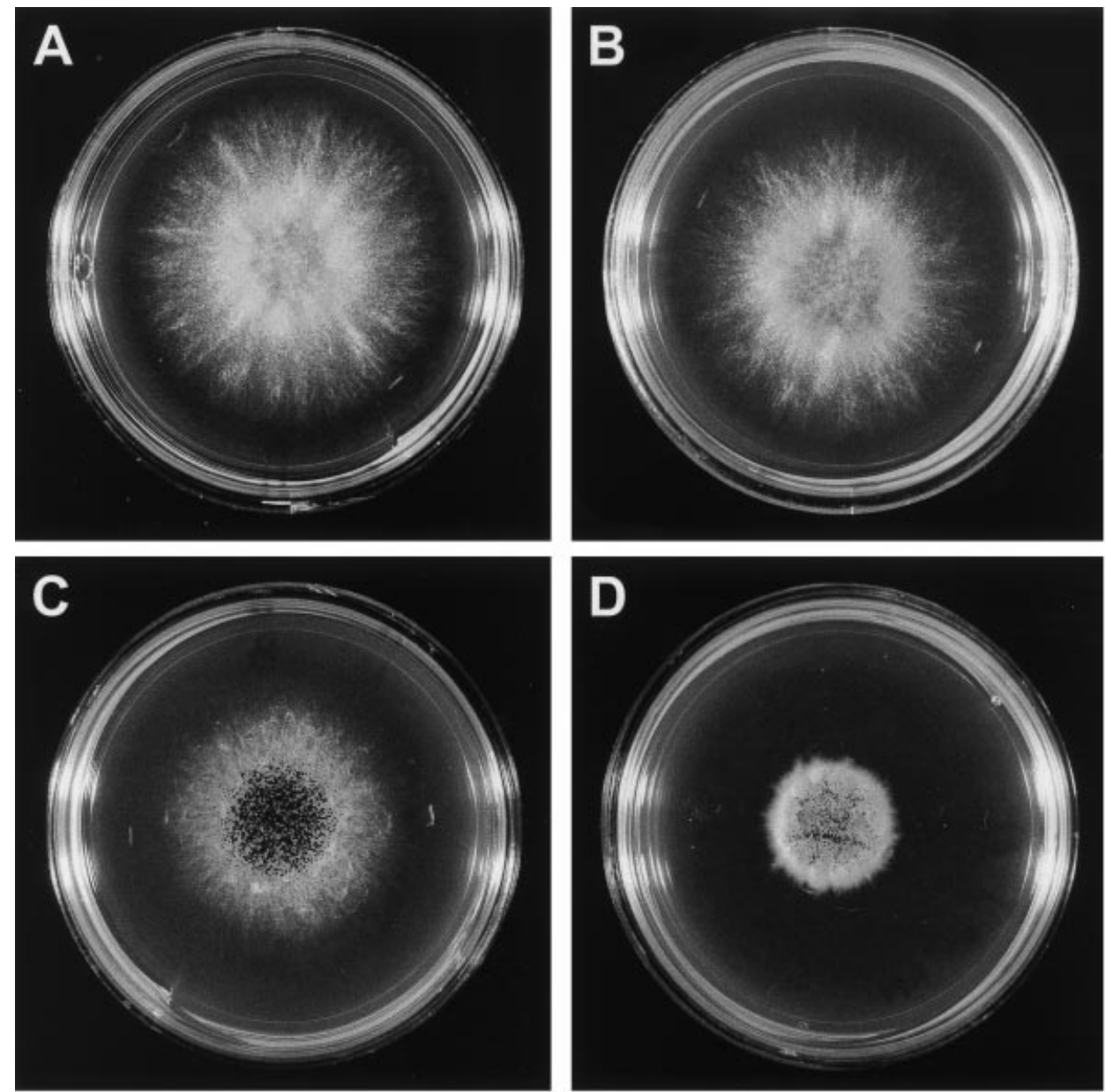

Fig. 1. Growth of $A$. niger and $A$. fumigatus after $48 \mathrm{~h}$ at $37{ }^{\circ} \mathrm{C}$ on ASP MM containing glucose, with and without $10 \mathrm{mM}$ CAMP. A. A. fumigatus, ASP MM. B. A. fumigatus, ASP MM+10 mM CAMP. C. A. niger, ASP MM. D. A. niger ASP $\mathrm{MM}+10 \mathrm{mM}$ CAMP.

the inhibitors were decreasing growth through another, non-PKA related mechanism. To demonstrate that the decreases in growth rate obtained with cAMP and PDE inhibitors were due to modulation of the cAMP/PKA pathway, rather than to non-specific toxicity, suboptimal concentrations of cAMP and IBMX were used in combination to show an additive effect on growth inhibition (Beebe \& Corbin, 1986). Indeed, on minimal medium plus glucose, the growth rate of $A$. niger was significantly inhibited by the combination $(P<0 \cdot 05)$ when compared with the growth rate on cAMP alone and IBMX alone (Table 2). In contrast, A. fumigatus did not display a significant change in growth rate on medium containing cAMP and IBMX when compared with both alone. Results with theophylline were similar to those obtained with IBMX (data not shown). These results support the interpretation that the decreased growth rate of A. fumigatus when grown in the presence of PDE inhibitors is due to toxicity or some other nonPKA related mechanism, whereas a PKA-specific mechanism appears to be active in $A$. niger.
Two lines of reasoning led us to investigate the role that carbon source might play on the growth response of $A$. fumigatus to cAMP. In S. cerevisiae, glucose is believed to act through a G-protein coupled receptor, Gpr1p, to cause a Gpa2p-dependent, transient increase in intracellular cAMP, followed by a longer-lived increase mediated through intracellular acidification-triggered RAS activation of adenyl cyclase (Kraakman et al., 1999). In A. niger growing on sucrose, intracellular acidification is also believed to lead to increased cAMP synthesis (Gradišnik-Grapulin \& Legiša, 1997). In both cases, the result is that the cAMP/PKA pathway is activated. Therefore, fructose, galactose, glycerol and acetate were chosen for testing, since they represent carbon sources that vary in their ability to be fermented and to participate in carbon catabolite repression. Fructose is a weakly repressing, fermentable carbon source; galactose is non-repressing and weakly fermentable; and glycerol and acetate are non-repressing and non-fermentable. When both A. niger and A. fumigatus were grown on fructose-containing medium with cAMP, 
Table 2. Growth rate $\left(\mu \mathrm{m} \mathrm{h}^{-1}\right)$ of $A$. fumigatus and $A$. niger when grown on glucose or glycerol minimal medium with CAMP and IBMX alone and in combination

\begin{tabular}{|c|c|c|}
\hline \multirow[t]{2}{*}{ Medium } & \multicolumn{2}{|c|}{ Growth rate } \\
\hline & A. fumigatus & A. niger \\
\hline \multicolumn{3}{|l|}{ ASP MM+glucose } \\
\hline Alone & $744 \pm 12$ & $702 \pm 6$ \\
\hline$+\mathrm{cAMP} *$ & $725 \pm 5$ & $453 \pm 10 \dagger$ \\
\hline$+0.5 \mathrm{mM}$ IBMX & $679 \pm 13+$ & $628 \pm 12 \dagger$ \\
\hline+ cAMP $^{*}$ and IBMX $\neq$ & $654 \pm 2 \dagger$ & $286 \pm 14 \mathbb{S}$ \\
\hline \multicolumn{3}{|l|}{ ASP MM + fructose } \\
\hline Alone & $692 \pm 2$ & $468 \pm 5$ \\
\hline$+\mathrm{cAMP} *$ & $682 \pm 14$ & $448 \pm 8 \dagger$ \\
\hline$+0.5 \mathrm{mM}$ IBMX & $676 \pm 5 \dagger$ & $436 \pm 6+$ \\
\hline$+\mathrm{cAMP}^{*}$ and IBMX‡ & $639 \pm 8+\$$ & $410 \pm 11+\$$ \\
\hline \multicolumn{3}{|l|}{ ASP MM + glycerol } \\
\hline Alone & $722 \pm 6$ & $533 \pm 1$ \\
\hline$+\mathrm{cAMP} *$ & $656 \pm 12 \dagger$ & $511 \pm 8$ \\
\hline$+0.5 \mathrm{mM}$ IBMX & $706 \pm 4$ & $479 \pm 10 \dagger$ \\
\hline$+\mathrm{cAMP}^{*}$ and IBMX‡ & $600 \pm 2+\$$ & $504 \pm 25+$ \\
\hline
\end{tabular}

* cAMP concentration for A. fumigatus, $10 \mathrm{mM}$; for A. niger, $1 \mathrm{mM}$ on glucose and $10 \mathrm{mM}$ on fructose and glycerol.

$+P<0.05$ compared to the growth control (alone).

$\ddagger$ IBMX concentration, $0.5 \mathrm{mM}$.

$\mathbb{S}$ Combination is significantly different from both cAMP and IBMX alone, $P<0 \cdot 05$.

Table 3. PKA activity, following CAMP addition, in cell extracts from $A$. fumigatus and $A$. niger grown on glucose and glycerol

\begin{tabular}{|c|c|c|c|c|}
\hline \multirow[t]{3}{*}{ Time $(\min )^{*}$} & \multicolumn{4}{|c|}{ Specific activity $\left[\mathrm{U}(\mathrm{mg} \text { protein })^{-1}\right]$} \\
\hline & \multicolumn{2}{|c|}{ A. fumigatus } & \multicolumn{2}{|c|}{ A. niger } \\
\hline & Glucose & Glycerol & Glucose & Glycerol \\
\hline 0 & $31 \cdot 7 \pm 0 \cdot 9$ & $6 \cdot 2 \pm 1 \cdot 3$ & $4 \cdot 2 \pm 1 \cdot 5$ & $10 \cdot 1 \pm 2 \cdot 1$ \\
\hline 5 & $26 \cdot 0 \pm 1 \cdot 8$ & $25 \cdot 8 \pm 2 \cdot 0 \dagger$ & $19 \cdot 6 \pm 0 \cdot 3+$ & $13 \cdot 3 \pm 3 \cdot 1$ \\
\hline 15 & $31 \cdot 5 \pm 2 \cdot 8$ & $9 \cdot 3 \pm 1 \cdot 6$ & $18 \cdot 0 \pm 0 \cdot 6+$ & $7 \cdot 5 \pm 3 \cdot 6$ \\
\hline 30 & $30 \cdot 2 \pm 0 \cdot 8$ & $5 \cdot 1 \pm 1 \cdot 6$ & $8 \cdot 8 \pm 0 \cdot 6$ & $1 \cdot 7 \pm 1 \cdot 0$ \\
\hline
\end{tabular}

* Time after addition of $10 \mathrm{mM}$ cAMP to culture.

† Significantly different from 0 time, $P<0 \cdot 05$.

only the growth of $A$. niger was significantly inhibited (Table 2). However, on galactose, glycerol and acetate, cAMP did significantly inhibit the growth rate of $A$. fumigatus, whereas the $A$. niger growth rate was inhibited on acetate (Tables 1 and 2). Indeed, on both fructose and glycerol, additive growth rate reduction was seen for A. fumigatus grown on a combination of cAMP and IBMX (Table 2). These data together support the idea that carbon catabolite repression may be involved in the response to exogenous cAMP in $A$. fumigatus.

\section{PKA activity}

To explore further the hypothesis that the insensitivity of A. fumigatus to the addition of exogenous cAMP may be due to the glucose activation of the cAMP/PKA pathway, kemptide phosphorylation was used to mea- 
sure PKA activity in cell extracts of A. fumigatus and $A$. niger. It had been previously shown that strains of $A$. niger in which the catalytic subunit of PKA is overexpressed, as a consequence of introduction of additional copies of the gene, exhibit increased kinase activity, as determined by kemptide phosphorylation (Bencina et al., 1997). A. niger was grown in minimal medium containing glucose and cAMP was added at time zero prior to cell harvest. Cell extracts from these cultures exhibited a 3.5-fold increase in kinase activity over the baseline at 5 and $15 \mathrm{~min}$ following cAMP addition, suggesting that PKA activity is increased by the addition of exogenous cAMP (Table 3). Under the same growth conditions, there was no change in the PKA activity of cell extracts from A. fumigatus, although the basal level of kinase activity for A. fumigatus was approximately sevenfold higher than that of $A$. niger (Table 3). The high basal level of activity of A. fumigatus may be near the organism's maximum, preventing further increases in activity stimulated by the addition of exogenous cAMP. This would be reflected in the failure to see a growth rate reduction in A. fumigatus on glucose medium with cAMP.

When the carbon source in the growth medium was changed to glycerol the kinase activity of A. fumigatus did respond to treatment with cAMP, showing a fourfold increase (Table 3). Interestingly, the kinase activity in A. niger extracts from cells grown on glycerol did not respond to the cAMP pulse. The difference in basal kemptide phosphorylation activity in extracts from A. fumigatus grown in glucose versus the basal activity in extracts from cells grown in glycerol supports the hypothesis that PKA activity may be maximal in medium containing glucose and, therefore, refractory to cAMP addition.

Both A. niger and A. fumigatus exhibited increases in kemptide phosphorylation activity under conditions in which they displayed reductions of growth. These data are consistent with the hypothesis that the cAMPinduced growth reduction is modulated through changes in carbon-source-regulated PKA activity. Although the precise mechanism underlying the carbon-source-dependent effect of cAMP remains to be elucidated, it raises interesting questions regarding the disparate regulation between these two Aspergillus species. The pathways and receptors used to sense glucose and other carbon sources may differ between the two species, which may be reflected in their different industrial roles. Although A. fumigatus is an important member of the compost cycle, it has little use in industrial processes whereas A. niger, which is cosmopolitan in distribution, is an important industrial source for citric acid and enzymes, such as amylase (Raper \& Fennell, 1965).

A. fumigatus responds to the addition of extracellular cAMP only when growing on a carbon source that is non-repressing. Our data supports the interpretation that during growth on glucose, the PKA activity is already near maximal, rendering the organism refractory to addition of exogenous cAMP. However, when basal levels of PKA are low, the organism responds to a pulse of cAMP, and PKA activity transiently increases. The results also suggest that $A$. fumigatus undergoes carbon catabolite repression in a manner similar to that of other fungi, and that glucose itself or intracellular acidification secondary to the metabolism of glucose results in activation of the cAMP/PKA pathway. The interrelationship between cAMP/PKA signalling and pathogenesis of other fungi and the recent finding that components of the pathway are up-regulated in a model of invasive aspergillosis, when coupled with the data presented here, suggest that PKA signalling is a fruitful area for continued study in A. fumigatus.

\section{ACKNOWLEDGEMENTS}

We would like to thank Jay Card for his photographic assistance. This work was supported in part by National Institutes of Health award AI041119 to J.C.R. and CA61909 to D.S.A.

\section{REFERENCES}

Beebe, S. \& Corbin, J. (1986). Cyclic nucleotide-dependent protein kinases. In The Enzymes, 3rd edn, vol. 17, pp. 43-111. Edited by P. Boyer \& E. Krebs. Orlando, FL: Academic Press.

Bencina, M., Panneman, H., Ruijter, G., Legiša, M. \& Visser, J. (1997). Characterization and overexpression of the Aspergillus niger gene encoding the cAMP-dependent protein kinase catalytic subunit. Microbiology 143, 1211-1220.

Casperson, G., Walker, N. \& Bourne, H. (1985). Isolation of the gene encoding adenylate cyclase in Saccharomyces cerevisiae. Proc Natl Acad Sci U S A 82, 5060-5063.

Cove, D. (1966). The induction and repression of nitrate reductase in the fungus Aspergillus nidulans. Biochim Biophys Acta 113, $51-56$.

D'Souza, C. \& Heitman, J. (2001). Conserved cAMP signaling cascades regulate fungal development and virulence. FEMS Microbiol Rev 3, 349-364.

D'Souza, C., Alspaugh, J., Yue, C., Harashima, T., Cox, G., Perfect, J. \& Heitman, J. (2001). Cyclic AMP-dependent protein kinase controls virulence of the fungal pathogen Cryptococcus neoformans. Mol Cell Biol 21, 3179-3191.

Gradišnik-Grapulin, M. \& Legiša, M. (1997). A spontaneous change in the intracellular cAMP level in Aspergillus niger is influenced by the sucrose concentration and by light. Appl Environ Microbiol 63, 2844-2849.

Kraakman, L., Lemaire, K., Ma, P., Tuenissen, A., Donaton, M., Van Dijck, P., Winderickx, J., de Winde, J. \& Thevelein, J. (1999). A Saccharomyces cerevisiae G-protein coupled receptor, Gpr1, is specifically required for glucose activation of the cAMP pathway during the transition to growth on glucose. Mol Microbiol 32, 1002-1012.

Kronstad, J., De Maria, A., Funnell, D., Laidlaw, R., Lee, N., de Sa, M. \& Ramesh, M. (1998). Signaling via cAMP in fungi: interconnections with mitogen-activated protein kinase pathways. Arch Microbiol 170, 395-404.

Kruger, J., Loubradou, G., Regenfelder, E., Hartmann, A. \& Kahmann, R. (1998). Crosstalk between cAMP and pheromone signaling pathways in Ustilago maydis. Mol Gen Genet 260, 193-198.

Lengeler, K., Davidson, R., D'Souza, C., Harashima, T., Shen, W., Wang, P., Pan, X., Waugh, M. \& Heitman, J. (2000). Signal 
transduction cascades regulating fungal development and virulence. Microbiol Mol Biol Rev 64, 746-785.

Malone, R. (1990). Dual regulation of meiosis in yeast. Cell 61, 375-378.

Raper, K. \& Fennell, D. I. (1965). The Genus Aspergillus. Baltimore, MD: William \& Wilkins.

Rhodes, J., Oliver, B., Askew, D. \& Amlung, T. (2001). Identification of genes of Aspergillus fumigatus up-regulated during growth on endothelial cells. Med Mycol 39, 253-260.

Shimizu, K. \& Keller, N. (2001). Genetic involvement of a cAMPdependent protein kinase in a $G$ protein signaling pathway regulating morphological and chemical transitions in Aspergillus nidulans. Genetics 157, 591-600.

Sonneborn, A., Bockmuhl, D., Gerads, M., Kurpanek, K., Sanglard, D. \& Ernst, J. (1999). Protein kinase A encoded by TPK2 regulates dimorphism of Candida albicans. Mol Microbiol 35, 386-396.

Taylor, S. \& Radzio-Andzelm, E. (1994). Cyclic AMP-dependent protein kinase. In Protein Kinases, pp. 2-29. Edited by J. R. Woodgett. Oxford: IRL Press.

Thevelein, J. (1994). Signal transduction in yeast. Yeast 10, 1753-1790.

Thevelein, J. M. \& de Winde, J. H. (1999). Novel sensing mechanisms and targets for the cAMP-protein kinase A pathway in the yeast Saccharomyces cerevisiae. Mol Microbiol 35, 904-918.

Toda, T., Cameron, S., Sass, P., Zoller, M., Scott, J., McMullen, B., Hurwitz, M., Krebs, E. \& Wigler, M. (1987). Cloning and characterization of $B C Y 1$, a locus encoding a regulatory subunit of the cyclic AMP-dependent protein kinase in Saccharomyces cerevisiae. Mol Cell Biol 7, 1371-1377.

Xu, J.-R., Urban, M., Sweigard, J. \& Hamer, J. (1997). The CPKA gene of Magnaporthe grisea is essential for appressorial penetration. Mol Plant Microbe Interact 10, 187-194.

Received 18 March 2002; accepted 10 April 2002. 